\title{
Elemene Nanoemulsion Inhibits Metastasis of Breast Cancer by ROS Scavenging
}

\author{
Bo $\mathrm{Han}^{1}$,* \\ Tao Wang $\mathbb{D}^{1, *}$ \\ Zhigang Xue ${ }^{2}$ \\ Tao Wen' \\ Ling $\mathrm{Lu}^{3}$ \\ Jie Meng' \\ Jian Liu (1D) \\ Sizhu $\mathrm{Wu}^{3}$ \\ Jianchun $\mathrm{Yu}^{2}$ \\ Haiyan Xu (D)
}

'Department of Biomedical Engineering, Institute of Basic Medical Sciences

Chinese Academy of Medical Sciences,

School of Basic Medicine Peking Union

Medical College, Beijing, People's

Republic of China; ${ }^{2}$ Department of

General Surgery, Peking Union Medical

College Hospital, Chinese Academy of

Medical Sciences \& Peking Union Medical

College, Beijing, People's Republic of

China; ${ }^{3}$ State Key Laboratory of Organic-

Inorganic Composites, Beijing University

of Chemical Technology, Beijing, People's

Republic of China

*These authors contributed equally to this work

\begin{abstract}
Introduction: Elemene $\left(\mathrm{C}_{15} \mathrm{H}_{24}\right)$ is a sesquiterpene compound extracted from the rhizome of Curcuma herbs. In the past decades, the anti-tumor activity of elemene has been observed in vitro and in some clinical practices. However, pharmacological mechanisms of elemene are not demonstrated adequately, which may lead to improper clinical applications. This study aimed to investigate the anti-tumor effect of elemene nanoemulsion in the mouse model of triple-negative breast cancer (TNBC) and reveal the underlying mechanisms.
\end{abstract}

Methods: The ESR measurement and quantum mechanics simulation were used to characterize the antioxidant ability of elemene nanoemulsion. The murine breast cancer cell line 4T1 cells were inoculated subcutaneously into the left fourth mammary fat pad of BalB/c mice to establish a TNBC mice model. The H\&E staining, immunohistochemical staining, DHE staining and Western blot were employed to evaluate the therapeutic effects of the elemene nanoemulsion on the TNBC mice.

Results: It was shown that the elemene nanoemulsion prolonged the survival of the triplenegative breast cancer-bearing mice and inhibited the metastasis to lung and liver while did not induce significant cytotoxicity to the tumor cells. Mechanistic studies demonstrated that the elemene nanoemulsion effectively scavenged the reactive oxygen species (ROS) in vitro and in vivo, which decreased the stabilization of hypoxia-inducible factor-1 $\alpha$ (HIF-1 $\alpha)$ and consequently reduced angiogenesis in the tumor microenvironment as well as decreased the level of NLRP3 inflammasomes and IL- $1 \beta$ production. In addition, the elemene nanoemulsion downregulated the level of IL-1 $\beta$ in the RAW264.7 cells in exposure with LPS.

Conclusion: In conclusion, due to the ROS scavenging ability, elemene nanoemulsion effectively inhibited the metastasis of the breast cancer cells to lung and liver and consequently prolonged the survival of TNBC mice.

Keywords: elemene, breast cancer, ROS, macrophages, tumor microenvironment

\section{Introduction}

Elemene $\left(\mathrm{C}_{15} \mathrm{H}_{24}\right)$ is a sesquiterpene compound extracted from the rhizome of Curcuma herbs. ${ }^{1}$ As its aqueous solubility is poor, elemene has been fabricated to nanoemulsion by combining with soybean phospholipids, cholesterol and sodium phosphates. ${ }^{2}$ In the past decades, most of the investigations consider the anti-tumor activity of the elemene nanoemulsion mainly comes from its mild cytotoxicity that is relatively lower than that of first-line chemotherapeutics, including inhibiting proliferation, arresting cell cycle and inducing apoptosis of cancer cells. ${ }^{3-7}$ For these reasons, the elemene nanoemulsion has been clinically applied to several types of cancers as an alternative chemotherapeutics for the patients who are too weak to endure conventional chemotherapies. In recent years, some investigations
Correspondence: Haiyan Xu; Jianchun Yu Email xuhy@pumc.edu.cn; yu-jch@I63. com 
observed that elemene could inhibit the invasion and metastasis of several types of tumor cells, for example, the elemene treatment reduced the metastatic nodules in lung of human gastric cancer cell xenograft nude mice, ${ }^{8}$ and inhibited the metastasis of mouse melanoma cell line B16F10 cells, ${ }^{2,9}$ human breast cancer cell line MCF-7 cells and MDA-MB-231 cells. ${ }^{10}$ Nevertheless, pharmacological mechanisms of the inhibitory effects are not adequately understood.

Over the past decades, it has been well recognized that tumor microenvironment contributes largely to the multiple-process of metastasis, and tumor cells can be normalized by modulating the microenvironment. ${ }^{11}$ Moreover, therapeutic strategies targeting to the tumor microenvironment may bring fewer side effects. Based on the existing observations that elemene could reduce the side effects of radio- and chemo-therapy ${ }^{12}$ and had the ability of antimetastasis, we hypothesized that the anti-tumor activity of elemene may come from its impacts upon the tumor microenvironment.

Breast cancer has become the most common cause of cancer death in women. ${ }^{13}$ Metastasis is the major cause of death in breast cancer, and the median survival for metastatic breast cancer is approximately 1 year. ${ }^{14}$ Triplenegative breast cancer (TNBC) is the most refractory one among all of the subtypes, for which hormone therapy and targeted therapy are unavailable because of the absence of estrogen receptor, progesterone receptor and human epidermal growth factor receptor $2 .{ }^{15}$ TNBC is usually highly invasive and has a higher rate of distant metastases with early recurrences, leading to a poorer prognosis compared to other subtypes. ${ }^{16}$ In this work, we chose mice TNBC as a model of microenvironment-driven metastasis to systematically investigate the anti-tumor effects and underlying mechanisms of elemene nanoemulsion from the view of tumor microenvironment modulation, aiming to provide an experimental basis for formulating more effective treatment regimens of elemene in the clinical application and also provide therapeutic options for metastatic TNBC.

\section{Materials and Methods}

\section{The Cell Culture}

The mouse breast cancer cell 4T1 cell line was purchased from Cell Bank of Shanghai Institutes of Biological Sciences, Chinese Academy of Sciences (Shanghai, China) and cultured in modified RPMI medium (Hyclone Laboratories, Logan, Utah, USA) supplemented with 10\% fetal bovine serum (FBS, Gibco, Life Technologies, Carlsbad, CA, USA), $100 \mathrm{U} / \mathrm{mL}$ penicillin and $100 \mu \mathrm{g} /$ $\mathrm{mL}$ streptomycin (Hyclone). And the mouse macrophage RAW 264.7 cell line was purchased from the Cell Resource Center of the Chinese Academy of Medical Sciences (Beijing, China) and cultured in DMEM high glucose medium (Hyclone) supplemented with 10\% FBS, $100 \mathrm{U} / \mathrm{mL}$ penicillin and $100 \mu \mathrm{g} / \mathrm{mL}$ streptomycin.

\section{The Characterization of Elemene Nanoemulsion}

The elemene nanoemulsion $(5 \mathrm{mg} / \mathrm{mL})$ was purchased from Dalian Huali Jingang Pharmaceutical co., ltd (H14022804, Dalian, China), and the major ingredients include Soybean phospholipids, cholesterol, ethanol, disodium hydrogen phosphate, and sodium dihydrogen phosphate. The size distribution of elemene nanoemulsion was measured by dynamic light scattering (DLS, Nano ZS90 Zetasizer, Malvern Instruments, Malvern, UK).

\section{Cell Viability Assay}

The cells were incubated with elemene at the dose of 10-80 $\mu \mathrm{g} / \mathrm{mL}$ for $24 \mathrm{~h}$. The cell viability was determined by CCK-8 (Dojindo Molecular Technologies, Kumamoto-ken, Japan). Cells were rinsed with PBS softly after incubation; then, 10 $\mu \mathrm{L}$ of CCK- 8 together with $100 \mu \mathrm{L}$ of fresh medium were added into the cells and incubated at $37^{\circ} \mathrm{C}$ for another 1 h. The optical density (OD) value at $450 \mathrm{~nm}$ was measured after incubation. Relative cell viability was calculated by normalizing the $\mathrm{OD}_{450}$ value to the control group.

\section{Apoptosis Assay}

Cells were incubated with elemene for $24 \mathrm{~h}$ before harvest. Apoptosis was determined using Annexin V-FITC Apoptosis Detection Kit (Dojindo Molecular Technologies). After incubation, the cells were rinsed twice with PBS and resuspended in $100 \mu \mathrm{L} 1 \times$ binding buffer. Then, cells were incubated with $5 \mu \mathrm{L}$ of Propidium iodide (PI) and $2.5 \mu \mathrm{L}$ of Annexin V-FITC for $15 \mathrm{~min}$ at room temperature in the dark before being subjected to a C6 Accuri flow cytometer (BD, USA).

\section{The Electron Spin Resonance (ESR) Spectroscopy}

The ESR measurements were carried out by a Bruker (Billerica, MA) EMX ESR spectrometer. Samples were added to a $1 \mathrm{~mm}$ internal diameter glass capillary tube. 
The 1, 1-Diphenyl-2-picryl-hydrazyl (DPPH), Superoxide radicals $\left(\mathrm{O}^{2 \cdot-}\right)$ and hydroxyl radicals $(\cdot \mathrm{OH})$ were used to assess the antioxidant capacity. Superoxide radicals $\left(\mathrm{O}^{\left.2 \cdot \cdot^{-}\right)}\right.$ and hydroxyl radicals $(\cdot \mathrm{OH})$ were generated through the xanthine/xanthine oxidase system and Fenton reaction, respectively. Testing parameters were set as follows: 20 $\mathrm{mW}$ microwave power, $1 \mathrm{G}$ field modulation, and 100 $\mathrm{G}$ scan range.

\section{The Quantum Mechanics Simulation}

The hydrogen dissociation energy of elemene was calculated using the quantum mechanics simulation method based on density functional theory. The elemene is fully geometrically optimized using generalized gradient approximation treated by the Perdew-Burke-Ernzerh of function of the exchange-correlation potential. The atomic orbital basis set Triple Numerical plus Polarization was used to calculate the external potential. The smearing value was set up in the self-consistent field procedure to improve the convergence quality. After the optimization of initial conformation, frequency analysis indicates no imaginary frequency. All detailed parameters were performed on the Dmol3 module using Materials Studio (MS) software (Accelrys Software Inc.).

The bond dissociation energy (BDE) value was calculated from the following equation: ${ }^{17}$

$$
\begin{aligned}
\Delta \mathrm{G}^{\mathrm{T}}(\mathrm{A}-\mathrm{H})=[\mathrm{E}(\mathrm{A} \bullet)+\mathrm{ZPVE}(\mathrm{A} \bullet)]+[\mathrm{E}(\mathrm{H} \bullet)+\mathrm{ZPVE}(\mathrm{H} \bullet)] \\
-[\mathrm{E}(\mathrm{AH})+\mathrm{ZPVE}(\mathrm{AH})]
\end{aligned}
$$

Where $\Delta \mathrm{G}^{\mathrm{T}}$ is the hydrogen dissociation energy at temperature $\mathrm{T}$ and the total energy is molecular energy $(\mathrm{E}-(\mathrm{AH}))$ and the corresponding radical energy $(\mathrm{E}-(\mathrm{A} \bullet)$ and $\mathrm{E}-(\mathrm{H} \bullet))$, the zero-point vibrational energy (ZPVE) represents the correction energy value at a certain temperature $\mathrm{T}$.

\section{The Measurement of Intracellular Reactive Oxygen Species (ROS)}

RAW264.7 cells were pre-treated with LPS $(50 \mu \mathrm{g} / \mathrm{L})$ in the presence or absence of IL-4 $(20 \mu \mathrm{g} / \mathrm{L})$ for 24 $\mathrm{h}$. Elemene was then added to the culture system and incubated for $24 \mathrm{~h}$. After incubation, Intracellular ROS was detected with 2'-,7'dichlorofluorescein diacetate (DCFH-DA, Sigma-Aldrich, Merck, Saint Louis, MO, United States). The cells were then washed with PBS and incubated with $10 \mu \mathrm{M}$ DCFH-DA for $30 \mathrm{~min}$ at $37^{\circ} \mathrm{C}$. After being washed, cells were resuspended in PBS and subjected to a flow cytometer immediately.

\section{The Quantitative Real-Time PCR}

RAW264.7 cells were treated as described above. Total RNA was extracted using TRIZOL (Sigma-Aldrich) and the concentration and quality were measured by NanoDrop ND-1000 (ThermoFisher). Total RNA was then reverse-transcribed into cDNA using PrimeScript ${ }^{\circledR}$ Reverse Transcriptase (Takara, Shiga, Japan). The mRNA levels of $I L-1 \beta$ and GAPDH were determined by qRT-PCR using SYBR ${ }^{\circledR}$ Premix Ex Taq ${ }^{\mathrm{TM}}$ reagent (Takara) on CFX 96 (BioRad). The Gapdh was taken as a loading control. The primers were as following:

Il- $1 \beta$ forward: CTTCAAATCTCGCAGCAGCACAT, Il- $1 \beta$ reverse: CAGCAGGTTATCATCATCATCCC, Gapdh forward: GGCAAATTCAACGGCACAGTCAA, Gapdh reverse: GACATACTCAGCACCGGCCTCAC.

\section{The Mice Model and Administration Regimen}

Female BALB/c mice (6 weeks old) were kept in the Experimental Animal Center at the Institute of Basic Medical Sciences, Chinese Academy of Medical Sciences (Beijing, China) under specific pathogen-free conditions for one week before injecting tumor cells.

To establish TNBC mice, $4 \mathrm{~T} 1$ cells $\left(1 \times 10^{6}\right.$ cells suspended in $100 \mu \mathrm{L}$ sterile PBS) were inoculated subcutaneously into the left fourth mammary fat pad of mice. To evaluate the pathological changes, the TNBC mice were randomized into five groups $(n=5)$ and administered intraperitoneally with elemene nanoemulsion at the dose of $1,5,10,20 \mathrm{mg} / \mathrm{kg}$ every day. Mice were sacrificed on day 28 post-inoculation. The tumor tissues, livers and lungs were collected and the lungs and livers were fixed in $4 \%$ paraformaldehyde, and the tumor tissues were fresh frozen under $-80^{\circ} \mathrm{C}$.

To evaluate the life span of TNBC mice after elemene administration, tumor-bearing mice were randomized into five groups $(\mathrm{n}=10)$, and the therapeutic regimen is shown in Table 1.

Table I Intraperitoneal Administration Protocols for Survival Experiment

\begin{tabular}{|l|c|c|}
\hline Group & $\begin{array}{c}\text { Dose } \\
\text { (mg/kg) }\end{array}$ & Administration Regime \\
\hline Control & \multicolumn{2}{|c|}{$100 \mu \mathrm{L}$ of saline } \\
\hline Elemene & 1 & One course of treatment: once every day for \\
& 5 & two weeks. After every course, stop for \\
& 10 & a week to begin the next course. \\
& 20 & \\
\hline
\end{tabular}




\section{The H\&E and IHC Staining}

Hematoxylin and eosin (H\&E) staining and immunohistochemical (IHC) staining were performed according to the standard protocols by Servicebio (Wuhan, China). IHC staining was conducted with primary antibodies of HIF$1 \alpha$ (Abcam, Cambridge, UK), CD31 (Abcam), IL-1 $\beta$ (Servicebio), NLRP3 (Servicebio) and Caspase-1 (Servicebio) at $4^{\circ} \mathrm{C}$ overnight, then incubated with HRPlabeled Goat Anti-Rabbit IgG $(\mathrm{H}+\mathrm{L})$ (Servicebio) for 50 min at room temperature. Diaminobenzidine (DAB) was used to visualize the antigen-antibody reaction. The slides were photographed by microscopy (Olympus BX53). The average integrated optical density (IOD) of HIF-1 $\alpha$, CD31, IL-1 $\beta$, NLRP3 and Caspase-1 in three or five randomly selected areas for each group were calculated using ImagePro Plus 6.0 (Media Cybernetics, Inc.).

\section{The Measurement of ROS Production in the Tumor Tissue}

The ROS in tumor tissue was detected by dihydroethidium (DHE) according to the standard protocols by Servicebio. Fresh frozen tumor tissues were embedded in optimal cutting temperature compound (OCT, Sakura) and cut into $5 \mu \mathrm{m}$-thick sections. The sections were incubated with DHE dye (Servicebio) at $37^{\circ} \mathrm{C}$ for $30 \mathrm{~min}$, followed by incubating with DAPI for $10 \mathrm{~min}$ after washing. The sections were mounted and sealed with an antifluorescence quenching agent. The slides were visualized by a fluorescence microscope. The mean fluorescence intensity (MFI) of DHE in 3 randomly selected areas for each group was calculated by Image Pro Plus.

\section{Western Blot}

Tumor tissues were ground in RIPA lysis buffer supplemented with protease inhibitors (Sigma-Aldrich) using a tissue homogenizer (Schneider Electric, Rueil, France). The dissolved protein was collected through centrifuging at $12,000 \mathrm{rpm}$ for $15 \mathrm{~min}$ at $4{ }^{\circ} \mathrm{C}$. Equal amounts of protein $(30 \mu \mathrm{g})$ were loaded onto $12 \%$ SDS-PAGE gel for separating and then transferred onto polyvinylidene difluoride membranes (PVDF, Millipore, Merck). The membranes were then blocked by $5 \%$ skim milk for $90 \mathrm{~min}$. The membranes were probed with primary antibodies against HIF-1 $\alpha$, CD31 and $\beta$-actin (CST) followed by HRPconjugated secondary antibodies against rabbit $\operatorname{IgG}$ and mouse IgG (Jackson ImmunoResearch, PA, USA). The immunocomplexes were visualized by a chemo- luminescence image analysis system (Tanon, Shanghai, China) with enhanced chemiluminescence (ECL) system (Millipore).

\section{Statistical Analysis}

All of the data were expressed as mean \pm standard error of the mean (SEM). The statistical significance of differences among groups was determined by one-way ANOVA, followed by the least significant difference (LSD) for multiple comparisons. Statistical difference of survival curve was assessed using the Log rank test (Mantel-Cox). All statistical analyses were performed using GraphPad Prism 8.0.

\section{Results}

\section{Elemene Prolonged the Survival of TNBC Mice}

The concentration of elemene nanoemulsion was $5 \mathrm{mg} /$ $\mathrm{mL}$, and the average diameter of the emulsion droplets was $7.9 \mathrm{~nm}$ determined by DLS (Figure 1A), inserted as the molecular structure of elemene. The therapeutic efficacy of elemene for TNBC mice was investigated to verify the anti-tumor activity of elemene. Results showed that elemene prolonged the survival of TNBC mice. The median survival ranged from 43.5 days to 48 days for TNBC mice treated with different doses of elemene, while that was 37 days for the control group (Figure 1B). The splenomegaly was observed in tumor-bearing mice compared with healthy mice. ${ }^{18}$ After 3-week treatment, the splenomegaly was alleviated by elemene (Figure 1C), the average weight of spleen was $0.71 \mathrm{~g}, 0.53 \mathrm{~g}, 0.48 \mathrm{~g}, 0.44 \mathrm{~g}$ in the TNBC mice of $1,5,10$ and $20 \mathrm{mg} / \mathrm{kg}$ elemene treated group, respectively, with reduction of $14 \%, 36 \%, 42 \%, 47 \%$ comparing to the control group, respectively. It was also noticed that the treatment did not affect the volume and weight of primary tumor in the TNBC mice (Figure 1D and $\mathrm{E}$ ), implying the prolonged survival and remission of splenomegaly could be independent of the cytotoxicity of elemene.

We next examined the cytotoxicity of elemene to mouse breast cancer cell 4T1. Results showed that the elemene at $20 \mu \mathrm{g} / \mathrm{mL}$ and the lower concentration did not affect the viability of $4 \mathrm{~T} 1$ cells (Figure 2A), while the higher concentration resulted in a significant inhibitory effect on the cell viability. The $\mathrm{IC}_{50}$ was calculated to be $40.42 \mu \mathrm{g} / \mathrm{mL}$. Correspondingly, elemene-induced apoptosis of $4 \mathrm{~T} 1$ cells, the apoptotic rates of the cells were $0.9 \%$, 
A

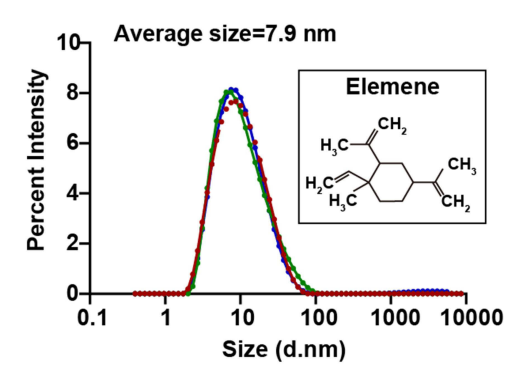

B

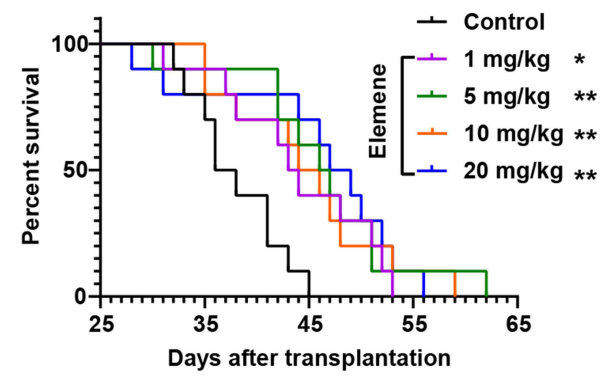

C

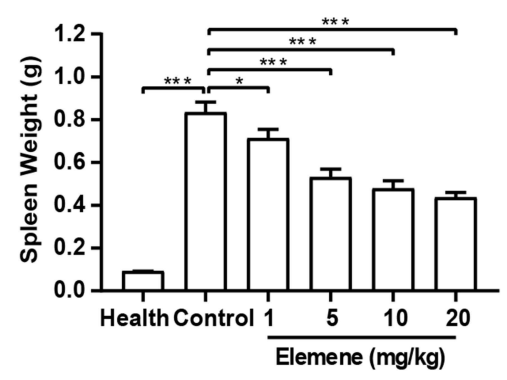

D

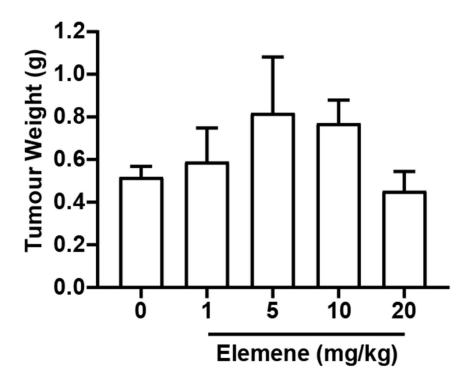

E

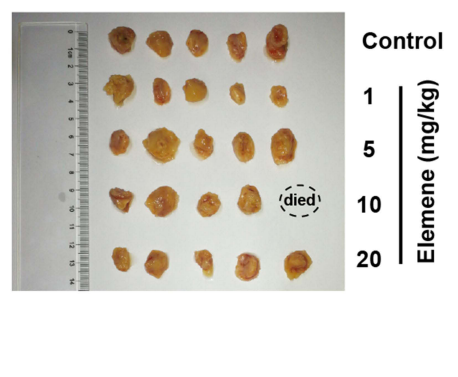

Figure I The administration of elemene nanoemulsion significantly prolonged the survival of TNBC mice. (A) Size distribution of the elemene nanoemulsion measured by DLS. (B) Survival curve of TNBC mice of control and elemene groups. Weights of spleens (C), images of tumor mass (D) and weights of tumor mass (E) obtained from TNBC-bearing mice after administration of elemene for 21 days. ${ }^{*} p<0.05$, ${ }^{* *} p<0.01$, ${ }^{* * *} p<0.001$.

A

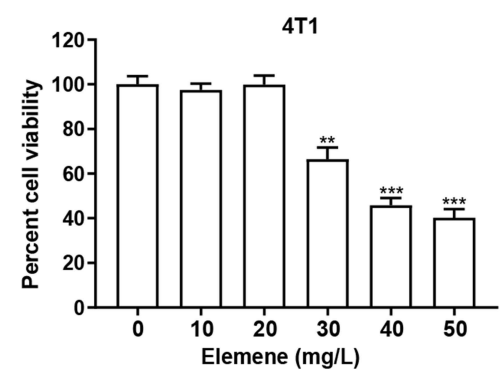

B

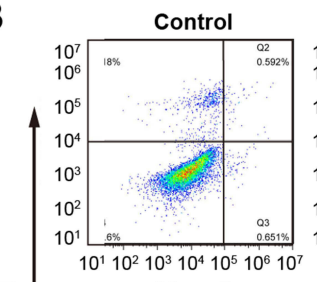

$\bar{\alpha}$

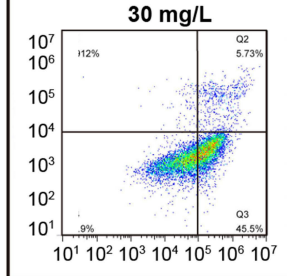

$10 \mathrm{mg} / \mathrm{L}$

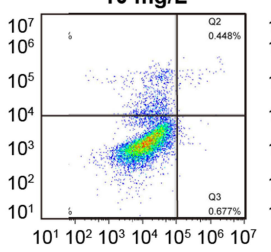
$40 \mathrm{mg} / \mathrm{L}$

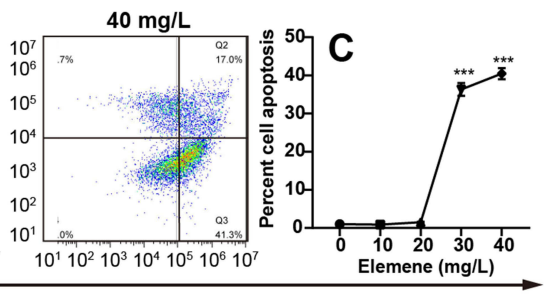

Annexin V-FITC

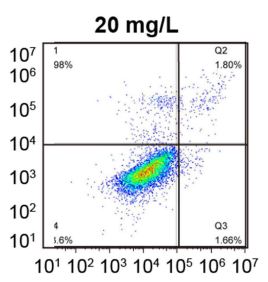

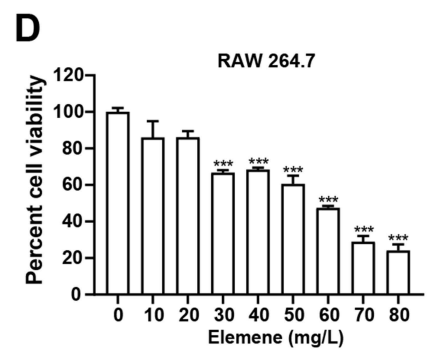
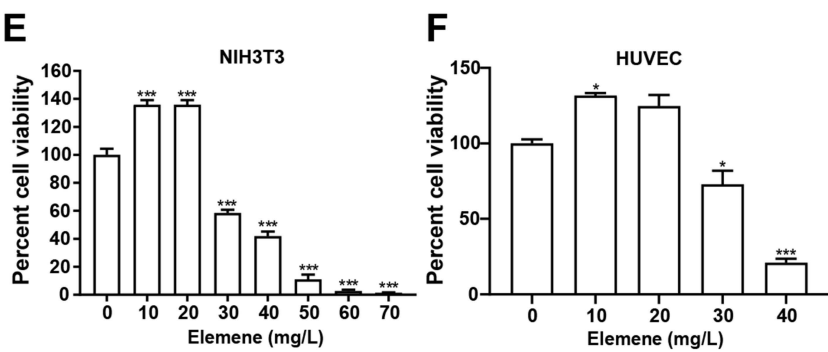

\section{G}

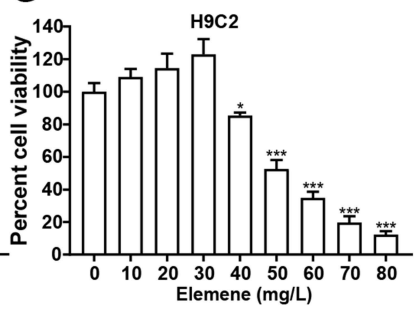

Figure 2 Elemene nanoemulsion showed a non-typical cytotoxic effect on cells. (A) The relative viability of 4 TI cells incubated with elemene for 24 h. (B) Representative scatter distribution for apoptosis staining of 4TI cells after incubated with elemene for $24 \mathrm{~h}$. (C) The statistical apoptotic rate of 4TI showed in Figure 2B. (D-G) The relative viability of NIH3T3, RAW 264.7, HUVEC and H9C2 incubated with elemene for $24 \mathrm{~h} .{ }^{*} \mathrm{p}<0.05, *^{*} \mathrm{p}<0.01$, **** $<$ < 0.001 .

$1.5 \%, 36.3 \%$ and $40.5 \%$ at $10,20,30$ and $40 \mu \mathrm{g} / \mathrm{mL}$ after incubation for $24 \mathrm{~h}$ (Figure 2B-C). It should be noted that there was a sudden drop in the cell viability when the dose reached $30 \mu \mathrm{g} / \mathrm{mL}$, suggesting elemene played different roles at different doses on the $4 \mathrm{~T} 1$ cells. The cytotoxicity of elemene to mouse fibroblast cells NIH3T3, mouse 
macrophages RAW264.7, human umbilical vein endothelial cells HUVEC, and rat cardiac myoblasts H9C2 was also examined by CCK8 assay. Results showed that the $\mathrm{IC}_{50}$ for RAW264.7, HUVEC, NIH3T3 and H9C2 was $34.05 \mu \mathrm{g} / \mathrm{mL}$ (Figure 2D), $51.85 \mu \mathrm{g} / \mathrm{mL}$ (Figure 2E), $34.92 \mu \mathrm{g} / \mathrm{mL}$ (Figure 2F), and $53.53 \mu \mathrm{g} / \mathrm{mL}$ (Figure $2 \mathrm{G}$ ), respectively, and the sudden drop of cell viability was also observed in these cells (Figure 2D-G). It could be noticed that for NIH3T3 and HUVEC, there was a slight increase in the cell viability when the elemene concentration was lower than $30 \mu \mathrm{g} / \mathrm{mL}$. Taken together, it was suggested that elemene is not a cytotoxic compound at relatively low concentrations.

\section{Elemene Inhibited TNBC Metastasis to Lung and Liver}

The growth and metastasis of $4 \mathrm{~T} 1$ cells in BABL/c mice resembles stage IV human breast cancer which is highly metastatic. ${ }^{19}$ Since lung and liver are the major target organs of $4 \mathrm{~T} 1$ cells, ${ }^{20}$ the metastasis in lung and liver tissues was examined by H\&E staining after 3-week administration of elemene. Obvious lung metastasis nodules (circled) were visualized in the control group, and elemene exhibited a dose-dependent suppressive effect in lung metastasis, exhibiting clear alveolar structure compared to the cell deposition in the control group (Figure 3A, upper column, lower magnification images could be found in Figure S1). The metastasized nodules counted macroscopically in the lung were also reduced by elemene (Figure 3B). Metastasis in the liver was also observed and pointed by arrows. It was shown that in the control group metastatic nodules appeared in hepatic parenchyma adjacent to the portal vein (arrowed in Figure 3A, lower column, lower magnification images could be found in Figure S2), and metastasis was inhibited by elemene, supported by the decrease in the statistics of metastatic nodules in the H\&E staining (Figure 3C).

A
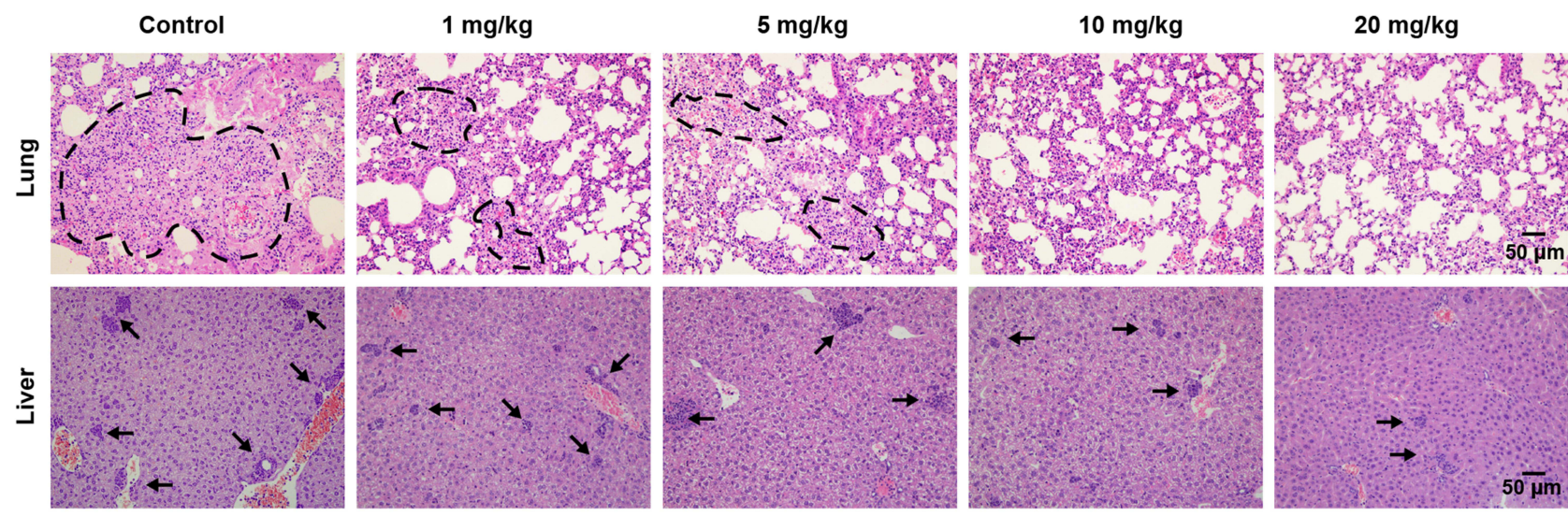

B

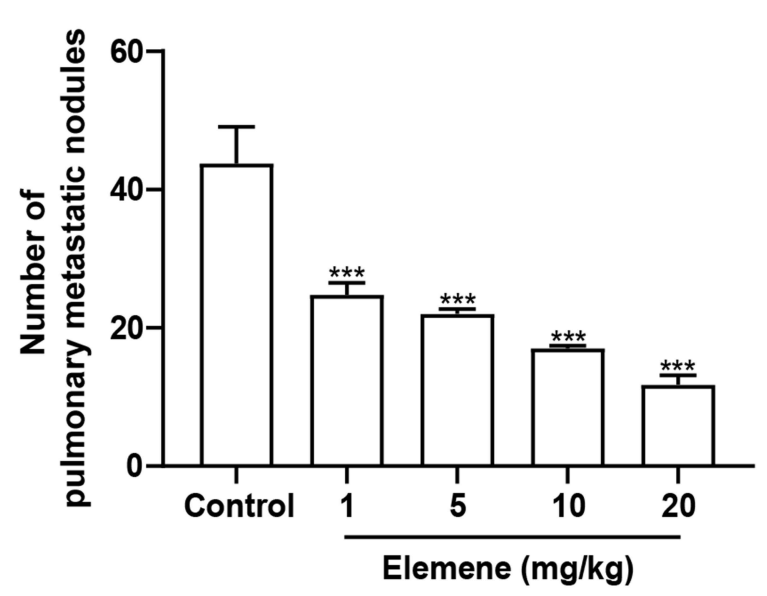

C

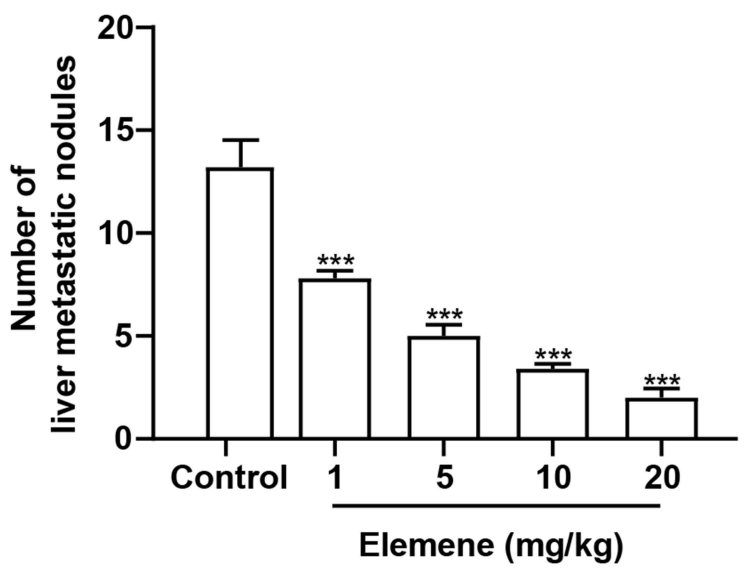

Figure 3 Elemene nanoemulsion inhibited metastasis to lungs and livers. (A) H\&E staining of lung and liver in the control and elemene groups. The scale bar indicated $50 \mu \mathrm{m}$, and the dashed lines and arrows pointed to the metastasis nodules in the H\&E staining images of lung and liver, respectively. (B) The number of metastatic nodules in the fresh lung. (C) The statistics of metastatic nodules in the liver in H\&E staining images. ${ }^{* * *} \mathrm{p}<0.00 \mathrm{I}$. 


\section{Elemene Showed ROS Scavenging Ability}

The above results drove us to think about the property and the anti-metastasis mechanism of elemene in cancer treatment. It can be noticed that there are 3 unsaturated bonds in every elemene molecule (Figure 1A, inserted), suggesting that elemene may have antioxidant ability. The ROS scavenging ability was measured by ESR spectroscopy. It was shown that elemene could reduce the signal intensity of both stable free radicals DPPH (Figure 4A) and active free radicals $\mathrm{O}_{2}{ }^{-{ }^{-}}$(Figure 4B) and $\cdot \mathrm{OH}$ (Figure $4 \mathrm{C}$ ) in a dose-dependent manner, which verified the ROS scavenging effects of elemene in vitro. To further clarify the ROS scavenging ability of elemene, the quantum mechanical simulation was performed. According to the hydrogen-atom transfer mechanism, the allylic hydrogen bond dissociation energy (BDE) is an important parameter for antioxidant efficiency. ${ }^{21}$ The hydrogen atom dissociation positions in the elemene structure are shown in Figure 4D, and the BDEs of elemene at these positions were calculated to evaluate the ability to scavenge radical efficiency (Figure 4E). The smaller BDEs were found at the position of (b) and (c), indicating that the allylic hydrogen bonds at (b) and (c) of elemene were readily broken to capture the radicals.

Then the ROS level within tumor tissue of TNBC mice who received 3-week administration of elemene was examined by DHE staining, and it was shown that the fluorescence of the elemene-treated group was weaker than that of the control group, indicating that the administration of elemene eliminated ROS in vivo (Figure 5A and $\mathrm{B})$.

\section{Elemene Reduced the Expression of HIF- $\mid \alpha$ and CD3I in Tumor Tissues}

Solid tumor cells proliferate rapidly and lead to the formation of a hypoxia area in the center of tumor tissue. ${ }^{22}$ In response to lower oxygen concentrations, a hypoxiainducible transcription factor HIF-1 $\alpha$ is activated and stabilized and played critical roles in progression and metastasis. ${ }^{23}$ ROS plays critical roles in the activation and stabilization of HIF- $1 \alpha,{ }^{24}$ therefore, it was reasonable to infer that elemene would downregulate HIF-1 $\alpha$ through ROS scavenging.
A

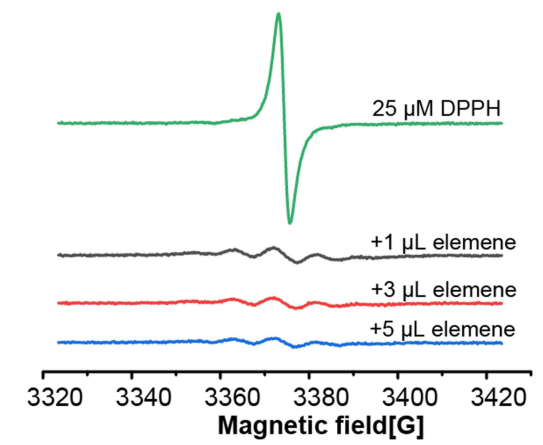

B

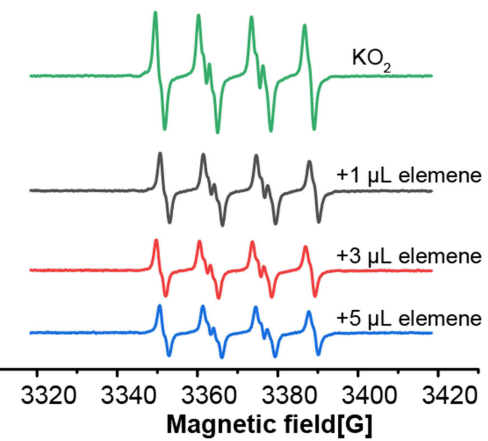

C

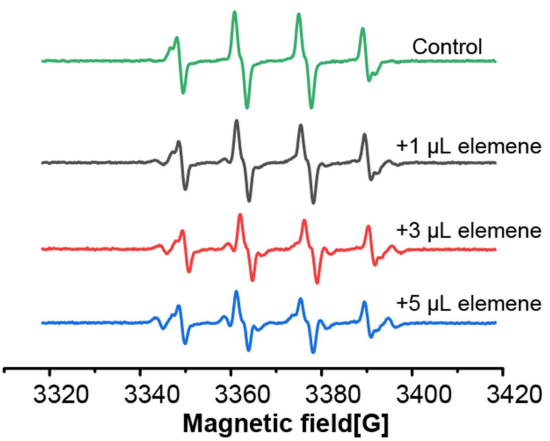

D

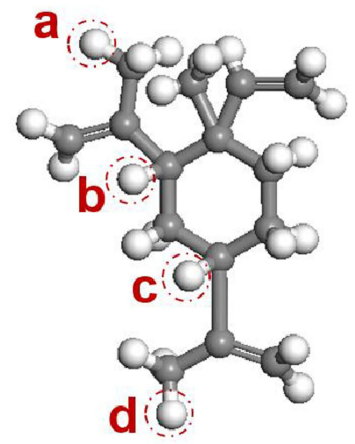

E

\begin{tabular}{cc}
\hline $\begin{array}{c}\text { Dissociation } \\
\text { positions }\end{array}$ & $\begin{array}{c}\text { Bond dissociation } \\
\text { energy (kJ/mol) }\end{array}$ \\
\hline a & 356.125 \\
b & 338.305 \\
c & 322.420 \\
d & 366.845 \\
\hline
\end{tabular}

Figure 4 Elemene showed ROS scavenging ability in vitro. (A-C) The ESR spectrum of DPPH, superoxide anion and hydroxyl radicals with or without elemene. (D) The hydrogen atom dissociation positions in elemene structure (Grey and white represent $\mathrm{C}$ and $\mathrm{H}$ atoms, respectively). (E) the BDEs of elemene at various positions. 
A

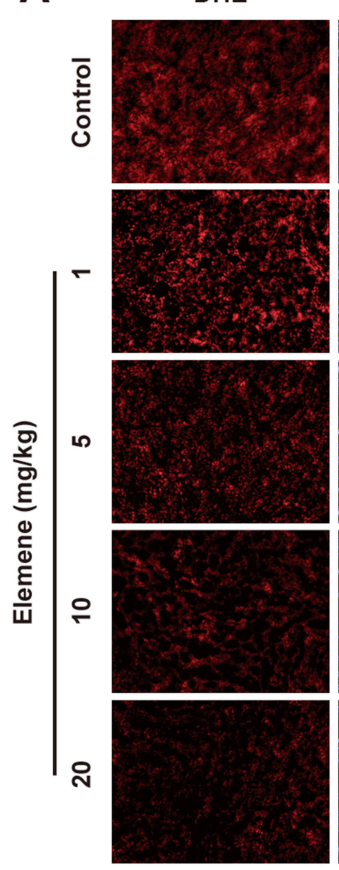

DAPI
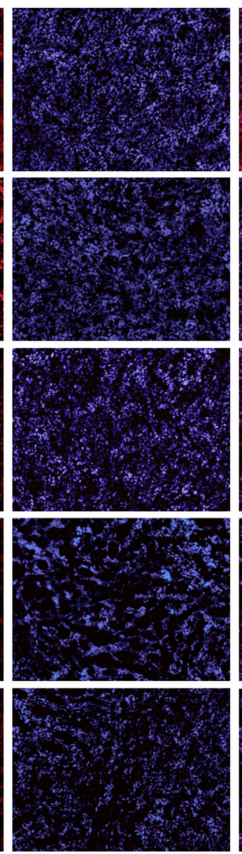
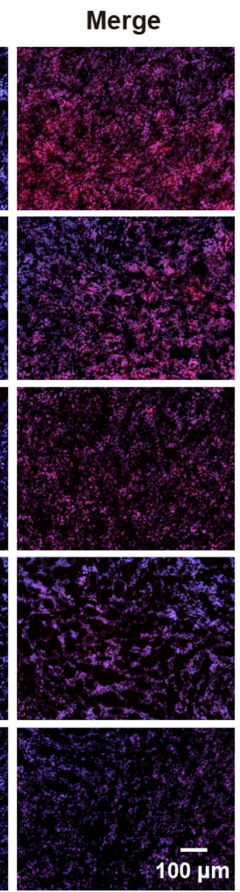

\section{Enlarge}

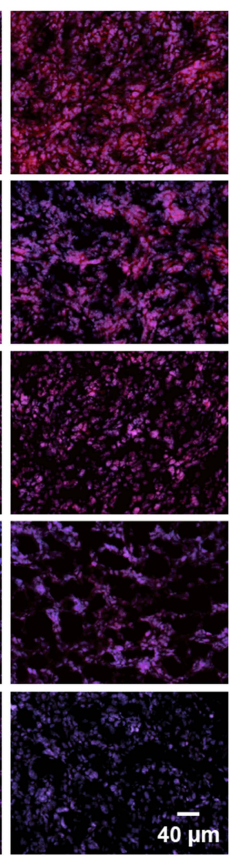

B

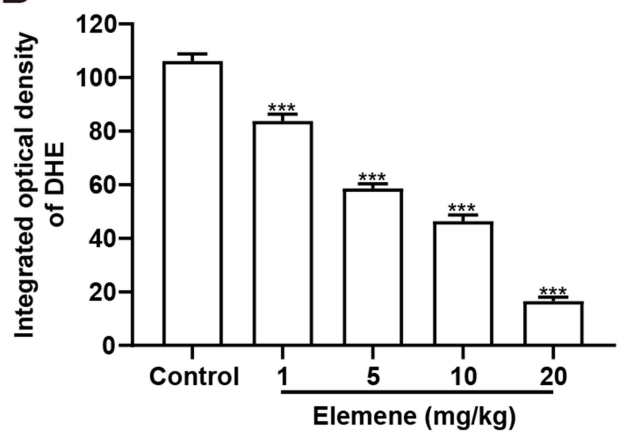

Figure 5 Elemene nanoemulsion eliminated ROS in vivo. (A) Representative images of DHE staining tumor tissues. The scale bar in the left 3 columns indicated I00 $\mu$ m, the scale bar in the right column indicated $40 \mu \mathrm{m}$. (B) The integrated optical density of DHE calculated by IPP. $* * *$ p $<0.00$ I vs control group.

To investigate the effect of elemene on HIF-1 $\alpha$ in vivo, tumor tissues were collected and subjected to IHC staining and Western blot. As shown, the IHC image of the control group is highly brown-colored (Figure 6A), suggesting that HIF-1 $\alpha$ was highly expressed in the untreated group. After administration of elemene, the expression of HIF-1 $\alpha$ was down-regulated in a dose-dependent manner evidenced by the decrease in the intensity of the brown-colored area (Figure 6A and B). Results of Western blotting were consistent with the IHC staining, which showed that the expression of HIF-1 $\alpha$ in tumor lysate of the elemene group was much lower than that of the control groups (Figure 6C and D). The results suggested that elemene could alleviate the hypoxia microenvironment in breast tumors.

Tumoral blood vasculature provides nutrition, oxygen and pathway for tumor growth and metastasis. ${ }^{25}$ The proliferation of tumor cells was supplied by nearby vessels aggravates hypoxia. ${ }^{26}$ Under hypoxia conditions, HIF- $1 \alpha$ stimulates transcription of angiogenesis-related genes to induce angiogenesis. ${ }^{27} \mathrm{CD} 31$ was expressed in endothelial cells and served as a representative molecule for tumor angiogenesis evaluation. ${ }^{28}$ The IHC staining showed a positive expression of CD31 in tumor tissues of the control group and the intensity was reduced by elemene (Figure 7A and B). Western blot analysis further verified that elemene decreased CD31 expression in tumor tissue (Figure 7C and D), suggesting that elemene inhibited angiogenesis in tumor tissues.

\section{Elemene Decreased Inflammatory Factors in vivo and in vitro}

TNBC harbors a specific inflammatory microenvironment which increases tumor tumorigenesis, invasion and metastases. ${ }^{29}$ Due to the relationship between ROS and inflammation, ${ }^{30}$ we then investigated the effect of elemene on inflammation in vivo and in vitro. It was well documented that IL-1 $\beta$ in tumor microenvironment act as a master proinflammatory cytokine in tumor progression, ${ }^{31}$ increased IL$1 \beta$ level was correlated with bad prognosis, carcinogenesis and invasiveness of the tumor. ${ }^{32}$ We found that the IL- $1 \beta$ expression was inhibited by elemene in vivo (Figure 8A, upper column, Figure 8B), evidenced by the decreased light density of the brown color in IHC staining. The production of IL-1 $\beta$ requires proteolytic cleavage by inflammasomes, which are multiprotein complexes that include NOD-like receptors, apoptosis-associated speck-like protein containing a caspase recruitment domain (ASC) and effector caspase$1 .^{33}$ NLRP3 is one of the most constitutively activated inflammasomes, which could be activated by $\operatorname{ROS}^{34}$ and could cleave the precursor pro-IL-1 $\beta$ into active IL- $1 \beta$ 

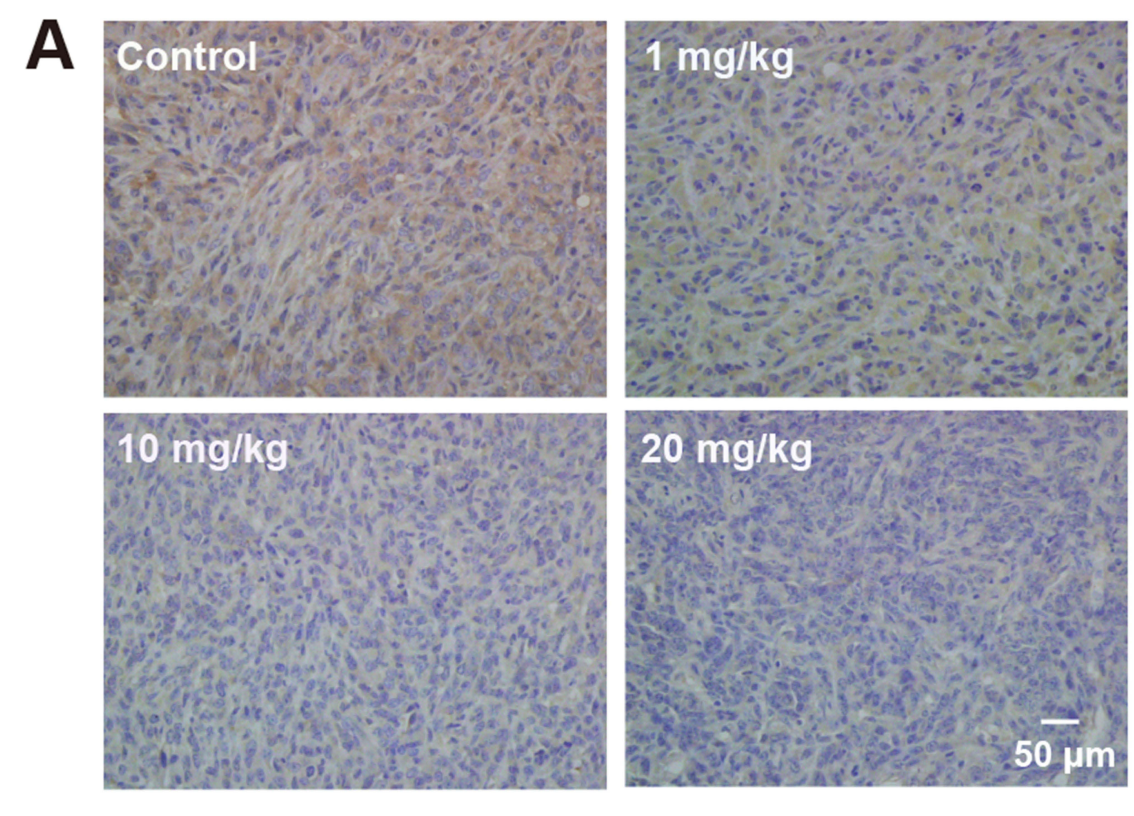

D
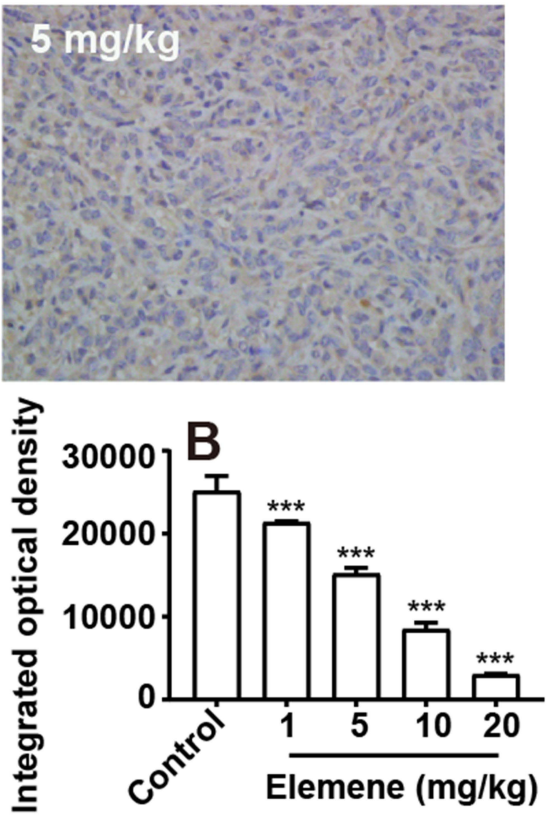
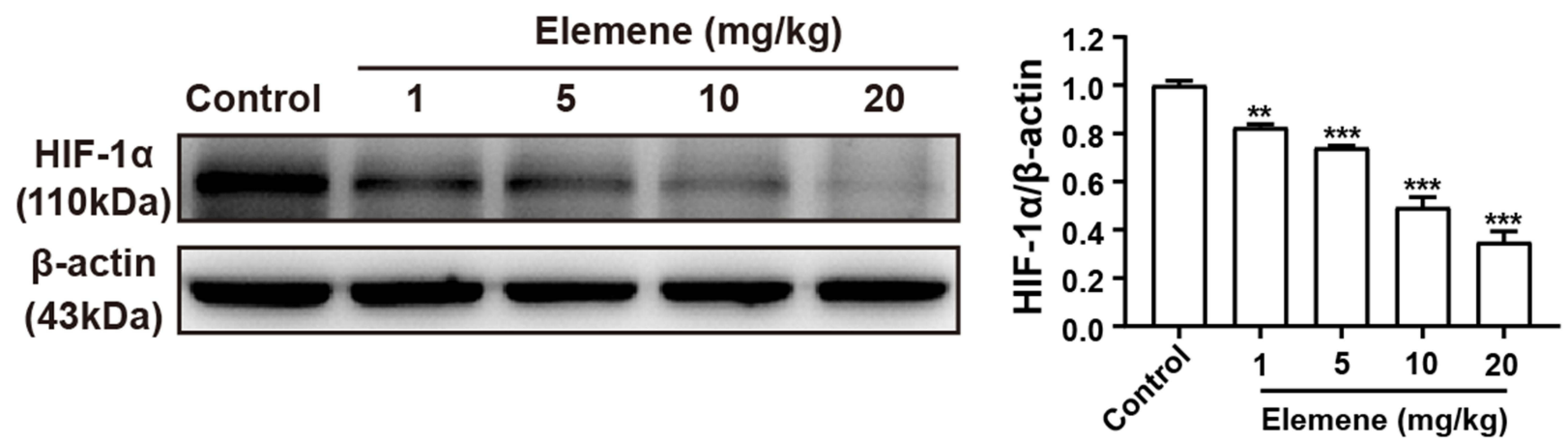

Figure 6 Elemene nanoemulsion downregulated HIF-I $\alpha$ in tumor tissues. (A) The immunohistochemical staining images of HIF-I $\alpha$ in control and elemene-treated groups. The scar bar indicated $50 \mu \mathrm{m}$. (B) The integrated optical density of HIF-I $\alpha$ in the immunohistochemical staining images. (C) Western blot analysis of HIF-I $\alpha$ in tumor lysate. (D) Quantitative analysis of lanes of Western blot. **p $<0.0$ I, ***p $<0.00$ I.

through caspase $1 .^{35}$ The NLRP3 and caspase-1 were highly expressed in the tumor tissues in the control group, while the NLRP3 and caspase-1 expression were eliminated by elemene treatment (Figure 8A, middle and lower column, Figure 8C and D), indicating that the elemene showed antiinflammation effect in vivo, which could be attributed to the ROS scavenging ability.

RAW264.7 cells were taken as a model to verify the anti-inflammatory effect in vitro. Results showed that elemene downregulated intracellular ROS of resting macrophages RAW264.7 and LPS activated inflammatory macrophages (Figure 9A), therefore downregulating the transcriptional level of IL-1 $\beta$ (Figure 9B). Moreover, in the breast cancer microenvironment, cancer cells may disable components of the immune system by secreting immunosuppressive factors and recruiting inflammatory cells that are actively immunosuppressive, leading to an immunosuppressive microenvironment. ${ }^{36}$ IL-4 was one of the most important cytokines involved in the immunosuppressive of breast cancer microenvironment. ${ }^{37}$ Here, IL-4 was used to mimic the immunosuppressive microenvironment in breast cancer. Under the immunosuppressive microenvironment, elemene also eliminated intracellular ROS and repressed the expression level of IL- $1 \beta$ (Figure 9C and D), these results together suggested the anti-inflammation effect of elemene in vitro.

\section{Discussion}

TNBC is characterized by a lack of indicated therapeutic targets and is highly metastatic; the primary therapeutic 

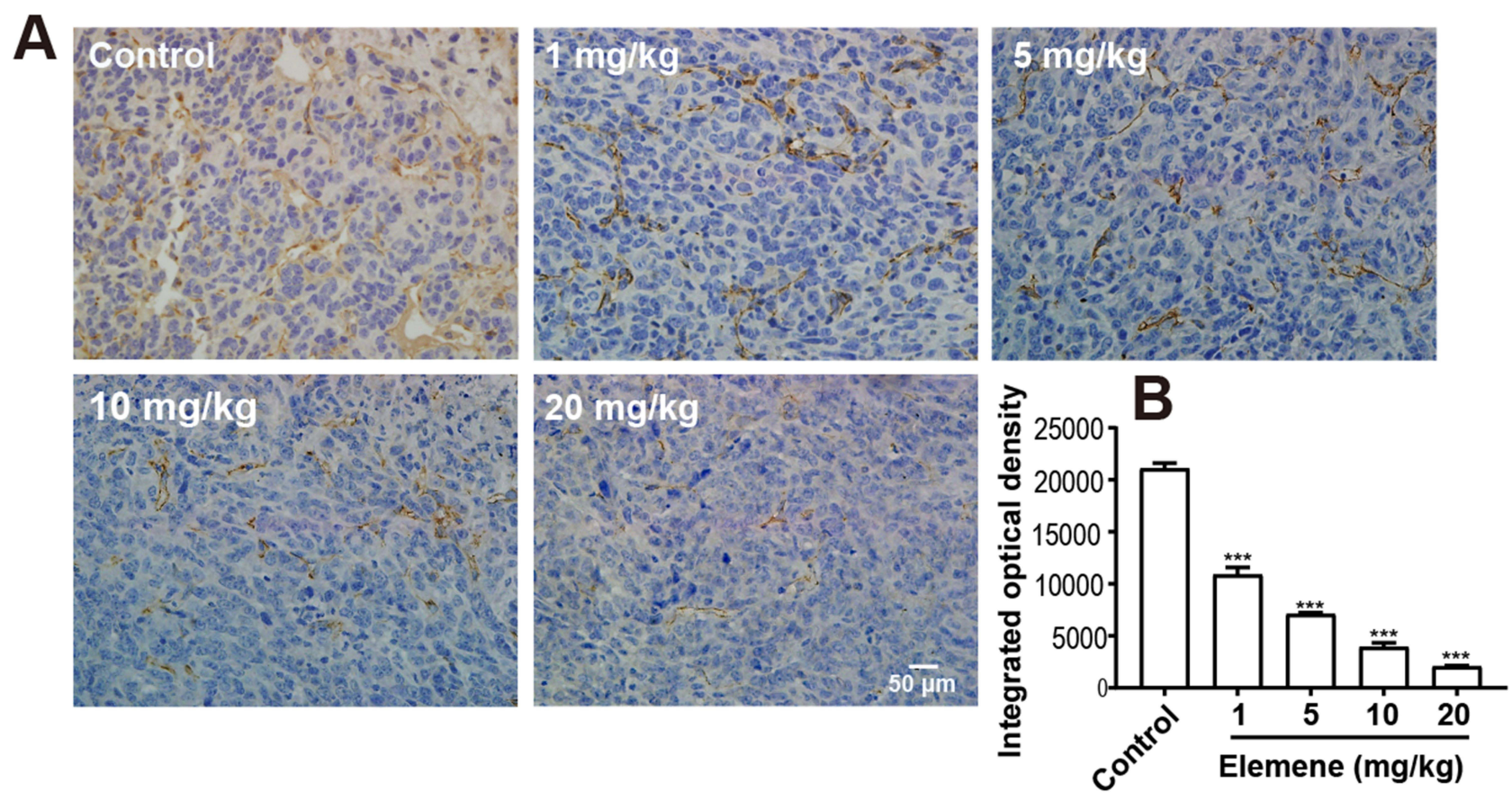

C

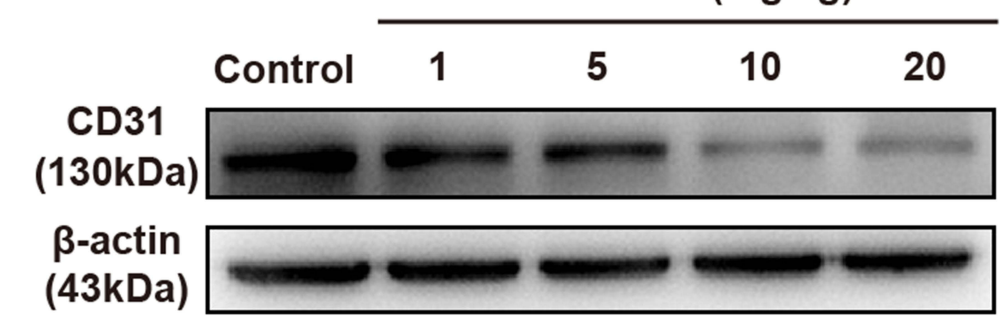

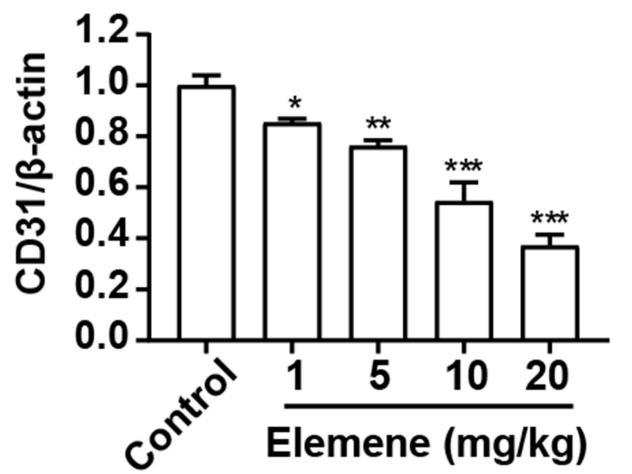

Figure 7 Elemene nanoemulsion reduced CD3I expression in primary breast tumor tissues. (A) The immunohistochemical staining of CD3I in control and elemenetreated groups. The scale bar indicated $50 \mu \mathrm{m}$. (B) The integrated optical density of CD3I in the immunohistochemical staining images. (C) Western blot analysis of CD3I in tumor lysate. (D) Quantitative analysis of lanes of Western blot. ${ }^{*} p<0.05, *^{*} p<0.01$, ***p $<0.001$.

option for TNBC is mainly conventional chemotherapy. ${ }^{14}$ As chemotherapy-related side effects are intolerable in elder patients or patients suffering from other diseases, elemene is used as an alternative therapeutics in clinical practices due to its relatively low toxicity compared to conventional chemotherapeutics to improve the quality of patients' life. ${ }^{38}$ However, the anti-tumor mechanism of elemene remains to be fully uncovered to benefit the clinical application. Our study revealed the antioxidant capacity of elemene from the view of chemical structure for the first time. By scavenging ROS, the elemene relieved the hypoxia and inflammatory status of the tumor microenvironment. On the basis of these results, one can design more effective regimens in the future, for example, combining elemene with an immunotherapeutic or cytotoxic chemotherapeutic.

Abundant macrophages infiltrate in breast tumors, accounting for up to $50 \%$ of tumor biomass, participating in tumor progression. ${ }^{39,40}$ Partial of the macrophages in the microenvironment continuously release pro-inflammatory factors to maintain the chronic inflammatory state of the tumor microenvironment and to promote tumor progression, ${ }^{41}$ while there are also some macrophages educated into tumor-associated macrophages, which construct an immunosuppressive environment and facilitate angiogenesis and metastasis. ${ }^{42}$ Although different subsets of macrophages 
A

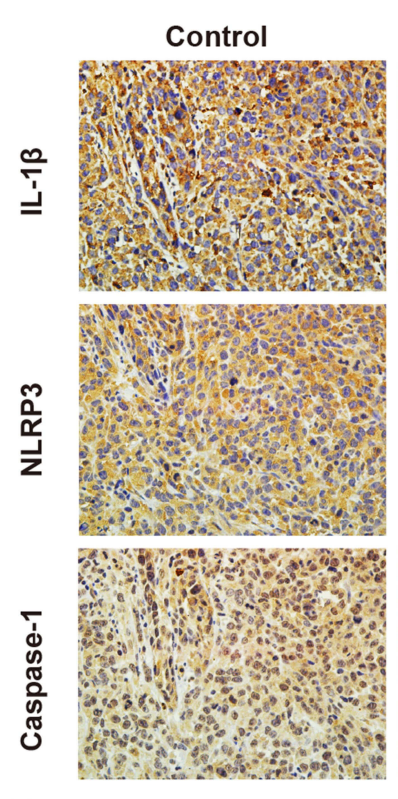

B

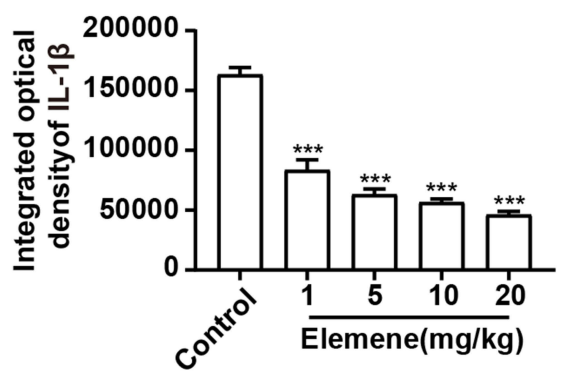

Elemene $(\mathrm{mg} / \mathrm{kg})$

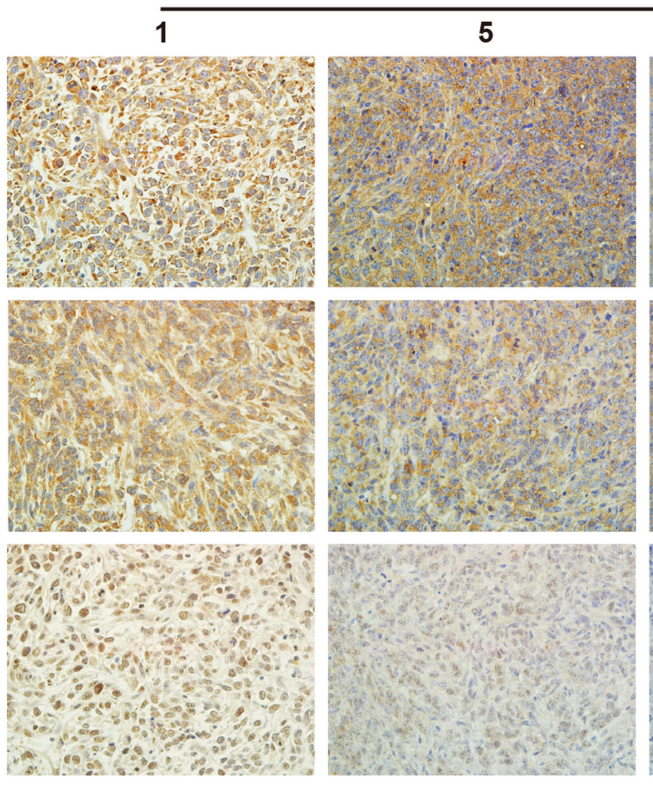

C

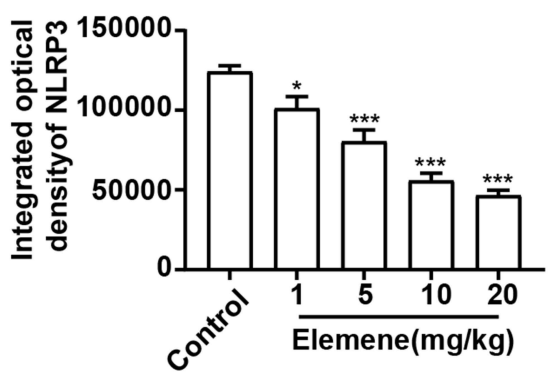

10
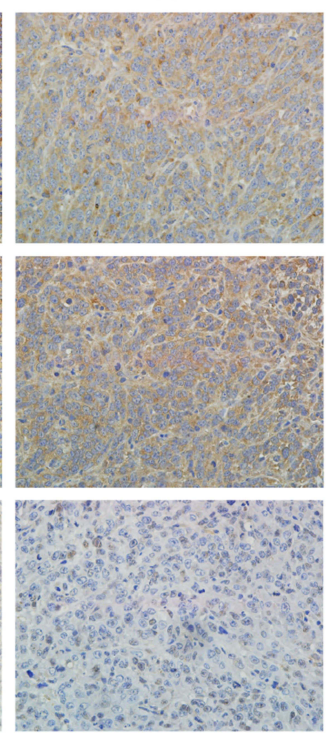

D

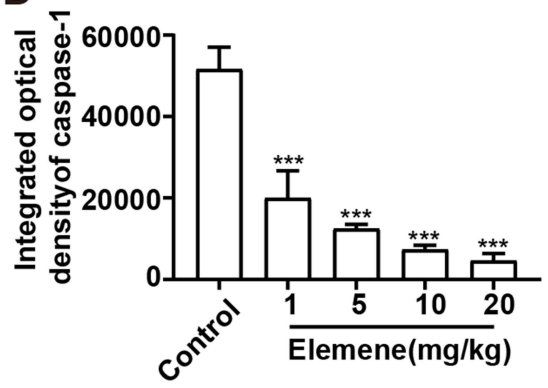

Figure 8 Elemene nanoemulsion decreased IL-I $\beta$, NLRP3 and caspase-I. (A) The immunohistochemical staining of IL-I $\beta$ (upper column), NLRP3 (middle column), and caspase-I (lower column) tumor tissues of the control and elemene-treated groups. The scale bar indicated $50 \mu \mathrm{m}$. (B-D) The integrated optical density of IL-I $\beta$, NLRP3 and caspase-I in the immunohistochemical staining images. ${ }^{*} \mathrm{p}<0.05$, $* * * p<0.00 \mathrm{I}$.

act differently in the tumor microenvironment, the increased macrophage infiltration is associated with advanced-stage disease and worse overall survival in breast cancer, ${ }^{43,44}$ providing a strong basis to target macrophages within the tumor microenvironment. In this study, it was shown that supplement of LPS or IL-4 aroused the intracellular ROS level of quiescent macrophages, resulting in secretion of inflammatory factors, which could be reversed by elemene, suggesting that elemene could reprogram the activated macrophages to resting ones. The macrophages-resting effect may be one of the reasons for the metastasis inhibition and survival prolongation.

Besides, ROS scavenging in macrophages showed potential to facilitate tumor immunotherapy. Immune checkpoint blockades, such as antibodies to programmed cell death-1 (PD-1) and its ligand PD-L1, are evolving treatment options for several types of cancer, but a large number of patients did not respond to immunotherapy. ${ }^{45}$ Preclinical studies suggested that the combination of PD1 or PD-L1 antibodies and cytotoxic chemotherapy could be a promising strategy for advanced cancer. However, it was documented that cytotoxic chemotherapy (such as paclitaxel) induced ROS, which increased PD-L1 expression in macrophages, PD-L1 positive macrophages have immune-suppressive and angiogenic properties that interfere with the efficacy of paclitaxel in vivo, ${ }^{46}$ and the ROS-inducing chemotherapy also weaken the efficacy of PD-L1 inhibitors. Unlike ROS-inducing paclitaxel, in this study, we found out that elemene scavenged intracellular ROS in macrophages, avoiding the potential tumor immune evasion caused by ROS production and PD-L1 overexpression. The ROS scavenging ability makes elemene an ideal option for the combination therapy with PD-L1 inhibitors. 

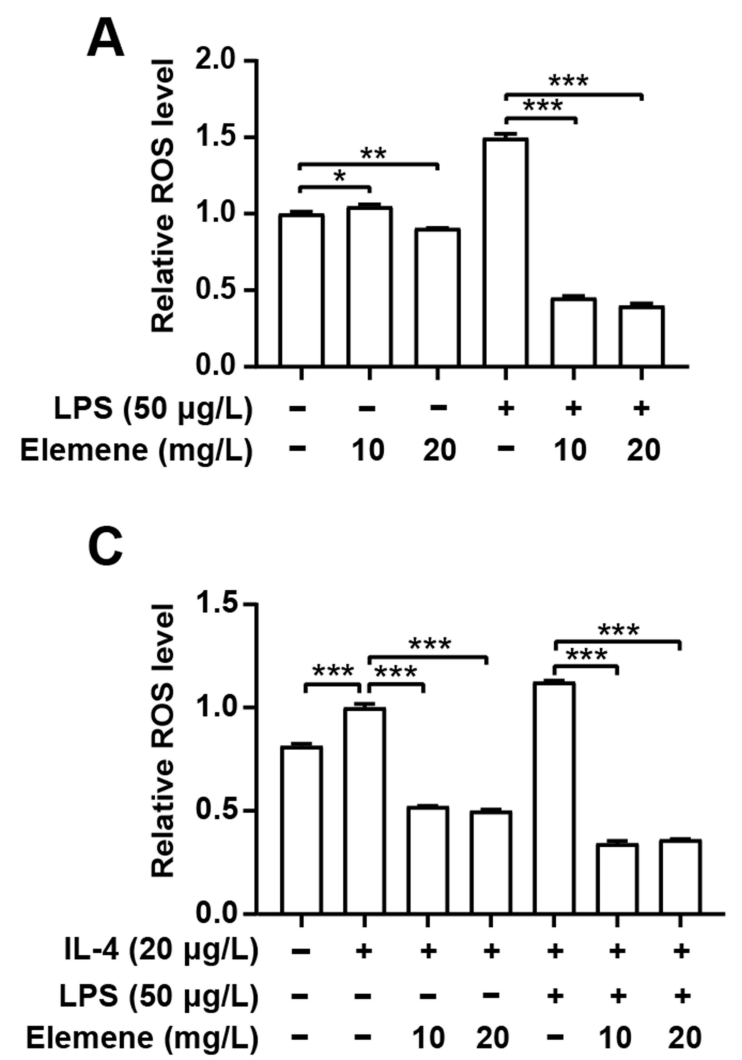
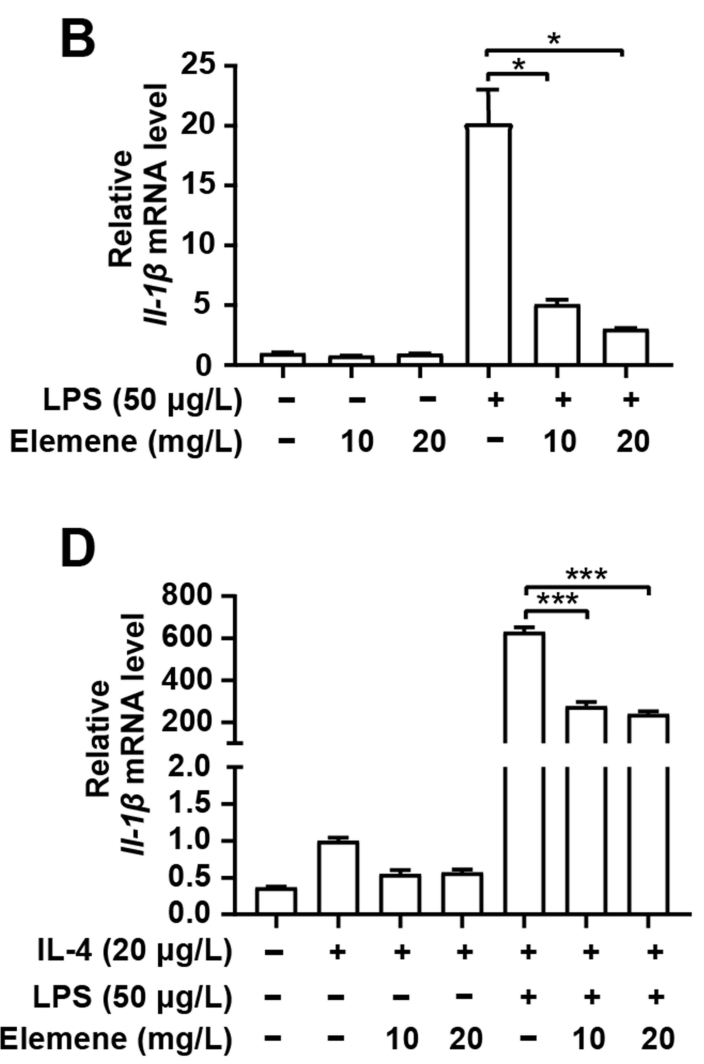

Figure 9 Elemene nanoemulsion down-regulated ROS and IL-I $\beta$ in macrophages RAW264 cells. (A) Intracellular ROS level of RAW264.7 cells after activation with LPS and then incubated with elemene. (B) Quantitative gene expression of IL-I $\beta$ in LPS-activated RAW264.7 treated with elemene. (C) Intracellular ROS level of RAW264.7 cells after activation with LPS in the presence of IL-4 and then incubated with elemene. (D) Quantitative gene expression of IL-I $\beta$ in LPS-activated RAW264.7 treated with elemene in the presence of IL-4. ${ }^{*} \mathrm{p}<0.05$, ${ }^{* *} \mathrm{p}<0.0 \mathrm{I}$, ${ }^{* * *} \mathrm{p}<0.00 \mathrm{I}$.

\section{Conclusion}

The elemene nanoemulsion significantly prolonged the survival of TNBC-bearing mice, the anti-tumor activity mainly came from its ROS scavenging ability that contributed to the inhibition of metastasis. Therefore, the elemene nanoemulsion is suggested to serve as a mild therapeutics to correct the tumor microenvironment while it should not be used as a cytotoxic chemotherapeutics.

\section{Ethics Approval}

This study was performed in accordance with the regulations of Chinese Academy of Medical Sciences Standing Committee on animal experiments and was approved by the Institutional Animal Care and Use committee (Institute of Basic Medical Sciences, Chinese Academy of Medical Sciences, and Peking Union Medical College, Beijing, China).

\section{Acknowledgment}

This work was supported by CAMS Innovation Fund for Medical Sciences (CIFMS, 2016-I2M-3-004) and the National Natural Science Foundation of China (51873017).

\section{Disclosure}

The authors report no conflicts of interest in this work.

\section{References}

1. Xie Q, Li F, Fang L, Liu W, Gu C. The antitumor efficacy of $\beta$ elemene by changing tumor inflammatory environment and tumor microenvironment. Biomed Res Int. 2020;2020:6892961. doi:10.11 $55 / 2020 / 6892961$

2. Chen W, Lu Y, Wu J, Gao M, Wang A, Xu B. Beta-elemene inhibits melanoma growth and metastasis via suppressing vascular endothelial growth factor-mediated angiogenesis. Cancer Chemother Pharmacol. 2011;67(4):799-808. doi:10.1007/s00280010-1378-x

3. Dai ZJ, Tang W, Lu WF, et al. Antiproliferative and apoptotic effects of $\beta$-elemene on human hepatoma HepG2 cells. Cancer Cell Int. 2013;13(1):27. doi:10.1186/1475-2867-13-27

4. Feng HB, Wang J, Jiang HR, et al. $\beta$-Elemene selectively inhibits the proliferation of glioma stem-like cells through the downregulation of notch1. Stem Cells Transl Med. 2017;6(3):830-839. doi:10.5966/ sctm.2016-0009

5. Wang G, Li X, Huang F, et al. Antitumor effect of beta-elemene in non-small-cell lung cancer cells is mediated via induction of cell cycle arrest and apoptotic cell death. Cell Mol Life Sci. 2005;62(78):881-893. doi:10.1007/s00018-005-5017-3

6. Wang L, Zhao Y, Wu Q, Guan Y, Wu X. Therapeutic effects of $\beta$ elemene via attenuation of the Wnt/ $\beta$-catenin signaling pathway in cervical cancer cells. Mol Med Rep. 2018;17(3):4299-4306. 
7. Wu J, Tang Q, Yang L, Chen Y, Zheng F, Hann SS. Interplay of DNA methyltransferase 1 and EZH2 through inactivation of Stat3 contributes to $\beta$-elemene-inhibited growth of nasopharyngeal carcinoma cells. Sci Rep. 2017;7(1):509. doi:10.1038/s41598-017-00626-6

8. Deng M, Liu B, Song H, et al. $\beta$-Elemene inhibits the metastasis of multidrug-resistant gastric cancer cells through miR-1323/Cbl-b/ EGFR pathway. Phytomedicine. 2020;69:153184. doi:10.1016/j. phymed.2020.153184

9. Shi H, Liu L, Liu LM, Geng J, Chen L. Inhibition of tumor growth by $\beta$-elemene through downregulation of the expression of uPA, uPAR, MMP-2, and MMP-9 in a murine intraocular melanoma model. Melanoma Res. 2015;25(1):15-21. doi:10.1097/CMR.000000 0000000124

10. Pan Y, Wang W, Huang S, et al. Beta-elemene inhibits breast cancer metastasis through blocking pyruvate kinase M2 dimerization and nuclear translocation. J Cell Mol Med. 2019;23(10):6846-6858. doi:10.1111/jcmm. 14568

11. Quail DF, Joyce JA. Microenvironmental regulation of tumor progression and metastasis. Nat Med. 2013;19(11):1423-1437. doi: $10.1038 / \mathrm{nm} .3394$

12. Shi Y, Chen T. Advances in anti-tumor effect and clinical application of elemene. Clin J Chin Med. 2020;12(18):40-45.

13. Available from: https://www.iarc.who.int/news-events/world-cancerday-2021/Accessed August 20, 2021.

14. Waks AG, Winer EP. Breast cancer treatment: a review. JAMA. 2019;321(3):288-300. doi:10.1001/jama.2018.19323

15. Yin L, Duan JJ, Bian XW, Yu SC. Triple-negative breast cancer molecular subtyping and treatment progress. Breast Cancer Res. 2020;22(1):61. doi:10.1186/s13058-020-01296-5

16. Vagia E, Mahalingam D, Cristofanilli M. The landscape of targeted therapies in TNBC. Cancers (Basel). 2020;12(4):916. doi:10.3390/ cancers 12040916

17. Sacks D, Baxter B, Campbell BCV, et al. Multisociety consensus quality improvement revised consensus statement for endovascular therapy of acute ischemic stroke. Int J Stroke. 2018;13(6):612-632.

18. DuPre SA, Hunter KW Jr. Murine mammary carcinoma 4T1 induces a leukemoid reaction with splenomegaly: association with tumor-derived growth factors. Exp Mol Pathol. 2007;82(1):12-24. doi:10.1016/j.yexmp.2006.06.007

19. Gilam A, Conde J, Weissglas-Volkov D, et al. Local microRNA delivery targets palladin and prevents metastatic breast cancer. Nat Commun. 2016;7(1):12868. doi:10.1038/ncomms 12868

20. DuPré SA, Redelman D, Hunter KW Jr. The mouse mammary carcinoma 4T1: characterization of the cellular landscape of primary tumours and metastatic tumour foci. Int $J$ Exp Pathol. 2007;88 (5):351-360. doi:10.1111/j.1365-2613.2007.00539.x

21. Guitard R, Paul JF, Nardello-Rataj V, Aubry JM. Myricetin, rosmarinic and carnosic acids as superior natural antioxidant alternatives to $\alpha$-tocopherol for the preservation of omega-3 oils. Food Chem. 2016;213:284-295. doi:10.1016/j.foodchem.2016.06.038

22. Brahimi-Horn MC, Chiche J, Pouyssegur J. Hypoxia and cancer. J Mol Med (Berl). 2007;85(12):1301-1307. doi:10.1007/s00109007-0281-3

23. Masoud GN, Li W. HIF-1alpha pathway: role, regulation and intervention for cancer therapy. Acta Pharm Sin B. 2015;5(5):378-389. doi:10.1016/j.apsb.2015.05.007

24. Aggarwal V, Tuli HS, Varol A, et al. Role of reactive oxygen species in cancer progression: molecular mechanisms and recent advancements. Biomolecules. 2019;9(11):735. doi:10.3390/biom9110735

25. Farnsworth RH, Lackmann M, Achen MG, Stacker SA. Vascular remodeling in cancer. Oncogene. 2014;33(27):3496-3505. doi:10.10 38/onc.2013.304

26. Voutouri C, Kirkpatrick ND, Chung E, et al. Experimental and computational analyses reveal dynamics of tumor vessel cooption and optimal treatment strategies. Proc Natl Acad Sci U S A. 2019;116(7):2662-2671. doi:10.1073/pnas.1818322116
27. Hashimoto T, Shibasaki F. Hypoxia-inducible factor as an angiogenic master switch. Front Pediatr. 2015;3:33. doi:10.3389/fped.2015. 00033

28. Baluk P, Morikawa S, Haskell A, Mancuso M, McDonald DM. Abnormalities of basement membrane on blood vessels and endothelial sprouts in tumors. Am $J$ Pathol. 2003;163(5):1801-1815. doi:10.1016/S0002-9440(10)63540-7

29. Sonnessa M, Cioffi A, Brunetti O, et al. NLRP3 inflammasome from bench to bedside: new perspectives for triple negative breast cancer. Front Oncol. 2020;10:1587. doi:10.3389/fonc.2020.01587

30. Hussain T, Tan B, Yin Y, Blachier F, Tossou MC, Rahu N. Oxidative stress and inflammation: what polyphenols can do for us? Oxid Med Cell Longev. 2016;2016:7432797. doi:10.1155/ 2016/7432797

31. Kaplanov I, Carmi Y, Kornetsky R, et al. Blocking IL-1 $\beta$ reverses the immunosuppression in mouse breast cancer and synergizes with antiPD-1 for tumor abrogation. Proc Natl Acad Sci U S A. 2019;116 (4):1361-1369. doi:10.1073/pnas.1812266115

32. Bent R, Moll L, Grabbe S, Bros M. Interleukin-1 beta-a friend or foe in malignancies? Int J Mol Sci. 2018;19(8):2155. doi:10.3390/ ijms 19082155

33. de Zoete MR, Palm NW, Zhu S, Flavell RA. Inflammasomes. Cold Spring Harb Perspect Biol. 2014;6(12):a016287. doi:10.1101/cshperspect.a016287

34. Zhou R, Yazdi AS, Menu P, Tschopp J. A role for mitochondria in NLRP3 inflammasome activation. Nature. 2011;469(7329):221-225. doi:10.1038/nature09663

35. Diakos CI, Charles KA, McMillan DC, Clarke SJ. Cancer-related inflammation and treatment effectiveness. Lancet Oncol. 2014;15 (11):e493-503. doi:10.1016/S1470-2045(14)70263-3

36. Hanahan D, Weinberg RA. Hallmarks of cancer: the next generation. Cell. 2011;144(5):646-674. doi:10.1016/j.cell.2011.02.013

37. Little AC, Pathanjeli P, Wu Z, et al. IL-4/IL-13 stimulated macrophages enhance breast cancer invasion via rho-GTPase regulation of synergistic VEGF/CCL-18 signaling. Front Oncol. 2019;9:456. doi:10.3389/fonc. 2019.00456

38. Jiang S, Ling C, Li W, Jiang H, Zhi Q, Jiang M. Molecular mechanisms of anti-cancer activities of $\beta$-elemene: targeting hallmarks of cancer. Anticancer Agents Med Chem. 2016;16(11):1426-1434. doi:10.2174/1871520616666160211123424

39. Pollard JW. Tumour-educated macrophages promote tumour progression and metastasis. Nat Rev Cancer. 2004;4(1):71-78. doi:10.1038/ nrc1256

40. Bingle L, Brown NJ, Lewis CE. The role of tumour-associated macrophages in tumour progression: implications for new anticancer therapies. J Pathol. 2002;196(3):254-265. doi:10.1002/path.1027

41. Qian BZ, Pollard JW. Macrophage diversity enhances tumor progression and metastasis. Cell. 2010;141(1):39-51. doi:10.1016/j. cell.2010.03.014

42. Laoui D, Movahedi K, Van Overmeire E, et al. Tumor-associated macrophages in breast cancer: distinct subsets, distinct functions. Int J Dev Biol. 2011;55(7-9):861-867. doi:10.1387/ ijdb.113371dl

43. Campbell MJ, Tonlaar NY, Garwood ER, et al. Proliferating macrophages associated with high grade, hormone receptor negative breast cancer and poor clinical outcome. Breast Cancer Res Treat. 2011;128 (3):703-711. doi:10.1007/s10549-010-1154-y

44. Poh AR, Ernst M. Targeting macrophages in cancer: from bench to bedside. Front Oncol. 2018;8:49. doi:10.3389/fonc.2018.00049

45. Hayashi H, Nakagawa K. Combination therapy with PD-1 or PD-L1 inhibitors for cancer. Int J Clin Oncol. 2020;25(5):818-830. doi:10.1007/s10147-019-01548-1

46. Roux C, Jafari SM, Shinde R, et al. Reactive oxygen species modulate macrophage immunosuppressive phenotype through the up-regulation of PD-L1. Proc Natl Acad Sci U S A. 2019;116 (10):4326-4335. doi:10.1073/pnas.1819473116 


\section{Publish your work in this journal}

The International Journal of Nanomedicine is an international, peerreviewed journal focusing on the application of nanotechnology in diagnostics, therapeutics, and drug delivery systems throughout the biomedical field. This journal is indexed on PubMed Central, MedLine, CAS, SciSearch ${ }^{\mathbb{R}}$, Current Contents ${ }^{\mathbb{R}} /$ Clinical Medicine, $^{2}$
Journal Citation Reports/Science Edition, EMBase, Scopus and the Elsevier Bibliographic databases. The manuscript management system is completely online and includes a very quick and fair peer-review system, which is all easy to use. Visit http://www.dovepress.com/ testimonials.php to read real quotes from published authors. 